\title{
Live birth following double-factor pre-implantation genetic diagnosis for both reciprocal translocation and alpha-thalassaemia
}

\author{
Vivian CY Lee *, Judy FC Chow, Estella YL Lau, William SB Yeung, Ernest HY Ng
}

\section{A B S T R A C T}

We report a live birth from a couple with two genetic diseases, namely: reciprocal translocation carrier and alpha-thalassaemia trait, following pre-implantation genetic diagnostic tests. This is the first case in Hong Kong in which the technique of using one blastomere biopsy for two diseases was established, using array comparative genomic hybridisation and polymerase chain reaction.
Hong Kong Med J 2014;20:251-4

DOI: $10.12809 / \mathrm{hkmj} 134087$

VCY Lee *, FHKAM (Obstetrics and Gynaecology)

JFC Chow, MPhil

EYL Lau, PhD

WSB Yeung, PhD

EHY Ng, MD

Department of Obstetrics and Gynaecology, The University of Hong Kong, Queen Mary Hospital, Pokfulam, Hong Kong

* Corresponding author: v200lee@hku.hk

\section{Introduction}

In this report, we present a couple who requested a double-factor pre-implantation genetic diagnosis (PGD) for both reciprocal translocation and alphathalassaemia.

\section{Case report}

Our patient, aged 36 years, enjoyed good past health and attended the subfertility clinic for recurrent miscarriage (5 times within 8 years). She had four spontaneous conceptions between 1997 and 2004 but all ended as first-trimester miscarriages. After 2004, she suffered from secondary subfertility and conceived again in 2007 following ovarian stimulation and intrauterine insemination. The fifth pregnancy again ended with first-trimester miscarriage.

She was subsequently referred to a clinical geneticist and found to be a carrier of a balanced reciprocal translocation 46,XX,t(2;10)(q33;q21.2). The husband had normal karyotypes and other relevant investigations for recurrent miscarriage were all negative. Both partners were alpha-thalassaemia trait carriers (South East Asia [SEA] type) as the genotype report revealed heterozygous alpha SEA type deletion. They were therefore referred to us for PGD.

Baseline investigations showed early follicular follicle-stimulating hormone levels of $6.5 \mathrm{IU} / \mathrm{L}$ and an antral follicle count of 19 . The couple was counselled about the procedure and risks of PGD. It was decided to biopsy two blastomeres, so as to perform polymerase chain reaction (PCR) for alpha-thalassaemia on one of them, and carry out fluorescent in-situ hybridisation (FISH) for the reciprocal translocation on the other.

The first cycle of in-vitro fertilisation (IVF) and intra-cytoplasmic sperm injection (ICSI) was carried out in November 2010. After 10 days of ovarian stimulation, 12 oocytes were retrieved and 11 were in metaphase II for ICSI. Ten were normally fertilised and seven day-3 embryos were available for embryo biopsy. Two embryos were subsequently shown to be normal for FISH signals and either normal or heterozygous for alpha-thalassaemia SEA deletion. On day 5 , there was only one fair-quality embryo at the morula stage for transfer, but the patient failed to conceive in that cycle.

She underwent a second IVF/ICSI/PGD cycle in January 2012. After 11 days of ovarian stimulation, 13 oocytes were retrieved. Twelve were fertilised, and eight day-3 embryos were available for embryo biopsy. One embryo was found to be balanced for the FISH signals and heterozygous for alphathalassaemia SEA deletion. That embryo developed to a good-quality blastocyst of grade $5 \mathrm{BB}$ and was transferred. She was pregnant but refused prenatal invasive testing in the second trimester because of the risk of miscarriage. She delivered a baby boy in October 2012 and the cord blood analysis confirmed the diagnosis of alpha-thalassaemia-1 carrier status with a normal karyotype 46,XY.

\section{Pre-implantation genetic diagnosis process \\ Optimisation process (direct mutation detection and linkage analysis)}

The Gap-PCR approach was used to amplify the alpha-SEA type deletion junction directly (Fig 1). 


\section{為平衡性轉位帶因者和甲型地中海負血兩種遺傳 基因疾病作胚胎植入前遺傳學診斷而結果為活產 的病例報告}

\author{
李芷茵、㱀鳳翔、劉綺蘭、楊樹標、吳鴻裕
}

本文報告一對患有平衡性轉位帶因者和甲型地中海貧血兩種遺傳基因 疾病的夫婦, 作胚胎植入前遺傳學診斷而活產的病例。這是香港首宗 病例, 我們亦發展了採用一個卵裂球活檢作兩種基因測試的技術（包 括陣列比較基因組雜交技術及聚合酶鏈反應）。
Briefly, the normal allele was amplified by primers Zdel-1 and Zdel-2 (317 bp), but not by Zdel-1 and Xdel-3, because they were too far apart. In the alpha SEA deletion, the binding site for Zdel-2 was deleted, and that for Zdel-1 and Xdel-3 were brought into close proximity producing a PCR product of $280 \mathrm{bp}$. Linkage analysis was performed with fluorescentlabelled intragenic informative markers (16pTEL05 and 16pTEL06) and linked short tandem repeats (STR) markers within $2 \mathrm{Mb}$ flanking the alpha-globin loci (D16S521 and D16S3395). The relative positions of primers and markers around the alpha SEA deletion are shown in Figure 1. Single cell protocols, using multiple displacement amplification (MDA) or SurePlex DNA amplification, have been validated using single lymphocytes from the couple.

\section{Embryo biopsy and pre-implantation genetic diagnosis}

Two blastomeres were biopsied from each of the good-quality day-3 embryos. One blastomere underwent whole genome amplification (WGA) and PCR for PGD on the alpha-thalassaemia loci. The second blastomere was lysed for translocation detection by FISH.

In the first PGD cycle, WGA was performed by the MDA method according to the protocol previously published. ${ }^{1}$ In the second cycle, SurePlex DNA amplification (BlueGnome) was adopted for

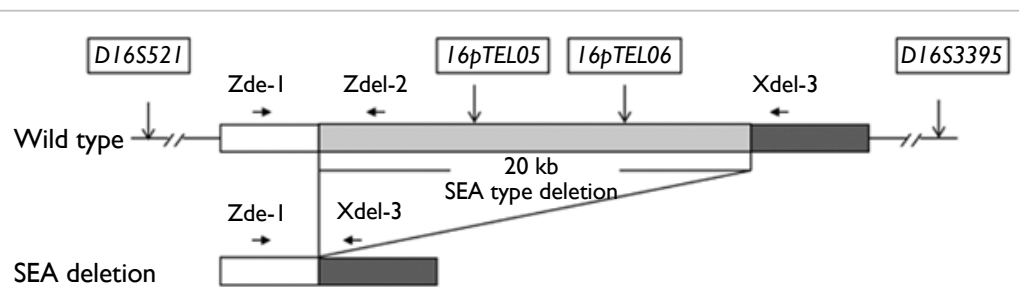

FIG I. Schematic diagram on the relative positions of Gap-polymerase chain reaction primers and microsatellite markers

Abbreviation: SEA = South East Asia type of alpha-thalassaemia trait
WGA. One $\mu \mathrm{L}$ of WGA product was used for PCR in a final volume of $25 \mu \mathrm{L}$ containing $1 \mathrm{X}$ PCR buffer with $\mathrm{MgSO}_{4}, 0.2 \mathrm{mM}$ dNTPs, and $1 \mathrm{U}$ FastStart Taq DNA polymerase (Roche). $0.5 \mu \mathrm{L}$ of PCR product was separated by an ABI 3500 genetic analyser with a GeneScan 500ROX-size standard (Applied Biosystems) and analysed by GeneMapper (v4.1; Applied Biosystems).

The second blastomere underwent FISH with Vysis probes Tel 2p (green), CEP10 (aqua), and Tel 10q (orange). The signals were interpreted independently by two scientists.

\section{Pre-implantation genetic diagnosis results}

In the first PGD cycle, Gap-PCR and intragenic informative markers 16pTEL05 and 16pTEL06 were used for PGD. All seven biopsied blastomeres resulted in a conclusive diagnosis. In the second cycle, the PGD protocol was modified in a few ways. Firstly, WGA was performed using the SurePlex DNA amplification system. Secondly, Gap-PCR primers Zdel-1 and Zdel-2 were omitted, since they were poorly amplified in SurePlex WGA DNA. Finally, two additional linked STR markers (D16S521 and D16S3395) were used to improve the diagnosis rate. Linkages of these additional markers with the SEA deletion locus were established with the leftover WGA DNA from embryos obtained in the first cycle. All embryos that underwent PGD showed conclusive results.

\section{Validation of one-blastomere protocol for double factor pre-implantation genetic diagnosis}

The leftover WGA DNA in the second cycle of PGD was used for array comparative genomic hybridisation (aCGH, 24Sure+, BlueGnome) for the detection of translocation. All samples showed conclusive result, which was consistent with those after FISH (Fig 2).

\section{Discussion}

Our unit has offered PGD treatment for monogenetic diseases for more than 10 years, starting in 2000 for alpha-thalassaemia. The FISH technique was then developed for translocation carriers and preimplantation genetic aneuploidy screening. In this case report, the couple described was the first to request PGD for both reciprocal translocation and alpha thalassaemia. The couple firstly attended our unit for PGD in 2009 and at that time the FISH technique was still routinely used for translocation. We decided to have two blastomeres biopsied, and undertook PCR on one (for alpha-thalassaemia) and FISH on the other (for reciprocal translocation). It is well-known that two-blastomere biopsy is more detrimental than one-blastomere biopsy on the implantation and pregnancy rate after embryo 
(a)
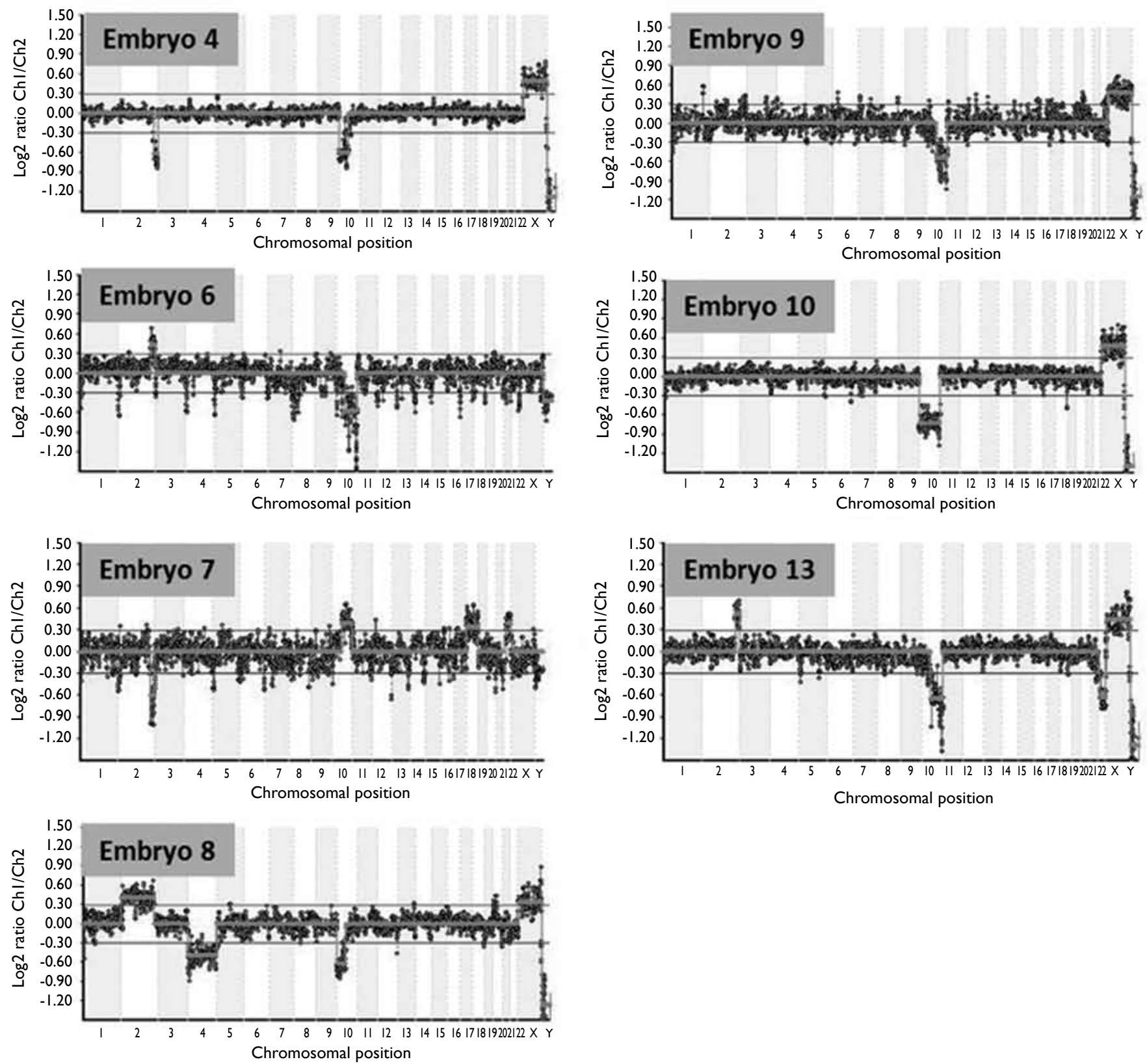

(b)

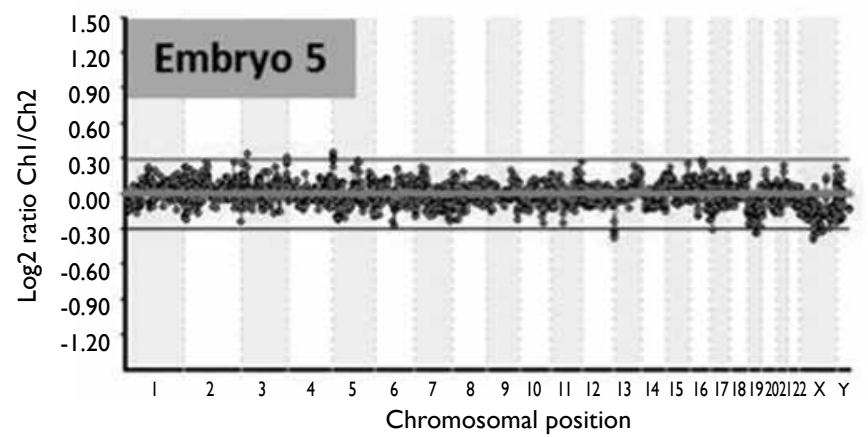

FIG 2. Array comparative genomic hybridisation result of (a) pre-implantation genetic diagnosis blastomeres; embryos 4, 6, 7, 8, 9, 10 , and I3 showing abnormal signals which are concordant with the fluorescent in-situ hybridisation (FISH) result, and (b) the embryo 5 (normal) which is replaced and results in a singleton live birth. Such result is concordant with both the FISH result and the karyotype of cord blood $(46, \mathrm{XY})$ 
transfer. ${ }^{2}$ Since 2008, there was emerging evidence regarding the use of array CGH in both translocation carriers and preimplantation aneuploidy screening. ${ }^{3-5}$ Using aCGH, it could obtain information on all 24 chromosomes to detect aneuploidy, which is common in early human embryos, other than in translocated genetic material. ${ }^{4}$ Recourse to WGA in aCGH allowed us to use a single blastomere for both diagnoses as the amplified products could also be used for PCR. We switched to using WGA with SurePlex. However, before we acquired the technique of aCGH for PGD of translocation in 2012, the couple requested the second treatment cycle because of advancing maternal age. Therefore FISH was used again in the second PGD cycle for translocation, as in the first cycle.

Later, we used the leftover WGA DNA from the second PGD cycle for translocation and aneuploidy detection, using aCGH 2 weeks after the PGD treatment cycles. All embryos with abnormal FISH signals showed abnormal aCGH results (Fig 2a). The normal embryo showed a normal signal with no aneuploidy detected after aCGH, which was performed immediately after the delivery of the baby boy (Fig 2b). Karyotyping on cord blood of the baby confirmed our PGD and aCGH results.

So far, there have been three case reports from the same group of investigators on the use of double-factor PGD. ${ }^{6-8}$ All of them involved couples at risk for one genetic disease only (cystic fibrosis, Von Hippel-Lindau syndrome, Lynch syndrome), but aneuploidy screening was performed to improve the implantation and pregnancy rates in those of advanced maternal age. Our patient was at risk for two genetic diseases, namely alpha-thalassaemia and reciprocal translocation. In the aforementioned case reports too, two cells were removed for PGD (either one polar body and one blastomere, or two blastomeres). Although we also had two blastomeres biopsied in the treatment cycle of our couple, we have validated a protocol with which doublefactor PGD can be performed with one-blastomere biopsy. With the use of aCGH, it becomes feasible and practicable to use one blastomere for both the monogenetic disease diagnosis and aCGH for either translocation carriers or aneuploidy screening in at-risk couples. The turnaround time of our protocol was approximately 2 days, rendering the fresh cycle day- 5 blastocyst feasible for transfer.

\section{Conclusion}

We report the first live birth after double-factor PGD for alpha-thalassaemia and reciprocal translocation. We have also validated a protocol for double-factor PGD, in which WGA DNA obtained from a single blastomere can be used for PCR-based PGD and aCGH.

\section{References}

1. Chow JF, Yeung WS, Lau EY, et al. Singleton birth after preimplantation genetic diagnosis for Huntington disease using whole genome amplification. Fertil Steril 2009;92:828. e7-10.

2. De Vos A, Staessen C, De Rycke $M$, et al. Impact of cleavage-stage embryo biopsy in view of PGD on human blastocyst implantation: a prospective cohort of single embryo transfers. Hum Reprod 2009;24:2988-96.

3. Wells D, Alfarawati S, Fragouli E. Use of comprehensive chromosomal screening for embryo assessment: microarrays and CGH. Mol Hum Reprod 2008;14:703-10.

4. Fiorentino F, Spizzichino L, Bono S, et al. PGD for reciprocal and Robertsonian translocations using array comparative genomic hybridization. Hum Reprod 2011;26:1925-35.

5. Forman EJ, Tao X, Ferry KM, Taylor D, Treff NR, Scott RT Jr. Single embryo transfer with comprehensive chromosome screening results in improved ongoing pregnancy rates and decreased miscarriage rates. Hum Reprod 2012;27:121722.

6. Obradors A, Fernández E, Oliver-Bonet M, et al. Birth of a healthy boy after a double factor PGD in a couple carrying a genetic disease and at risk for aneuploidy: case report. Hum Reprod 2008;23:1949-56.

7. Obradors A, Fernández E, Rius M, et al. Outcome of twin babies free of Von Hippel-Lindau disease after a doublefactor preimplantation genetic diagnosis: monogenetic mutation analysis and comprehensive aneuploidy screening. Ferti Steril 2009;91:933.e1-7.

8. Daina G, Ramos L, Obradors A, et al. First successful double-factor PGD for Lynch syndrome: monogenic analysis and comprehensive aneuploidy screening. Clin Genet 2012;84:70-3. 